\title{
Roles of the human microbiome in cancer
}

\author{
Toni Gabaldón ${ }^{1,2,3}$ \\ ${ }^{1}$ Barcelona Supercomputing Centre (BCS-CNS), Barcelona, Spain; ${ }^{2}$ Institute for Research in Biomedicine (IRB), The Barcelona Institute of Science \\ and Technology (BIST), Barcelona, Spain; ${ }^{3}$ Institució Catalana de Recerca i Estudis Avançats (ICREA), Barcelona, Spain \\ Correspondence to: Toni Gabaldón. Barcelona Supercomputing Centre (BCS-CNS). Jordi Girona, 29. 08034 Barcelona, Spain; Institute for Research in \\ Biomedicine (IRB), The Barcelona Institute of Science and Technology (BIST). Barcelona, Spain; Institució Catalana de Recerca i Estudis Avançats \\ (ICREA), Pg. Lluís Companys 23, 08010 Barcelona, Spain. Email: toni.gabaldon.ben@gmail.com. \\ Comment on: Sepich-Poore GD, Zitvogel L, Straussman R, et al. The microbiome and human cancer. Science 2021;371:eabc4552.
}

Submitted Jun 15, 2021. Accepted for publication Jun 28, 2021.

doi: 10.21037/hbsn-21-241

View this article at: https://dx.doi.org/10.21037/hbsn-21-241

All external surfaces and internal mucosae of the human body are inhabited by rich microbial ecosystems, collectively known as the human microbiome. These microbial communities, formed by bacteria, archaea, fungi, protozoa and viruses interact among them and with our own physiology in ways that we are still far from understand. However it is increasingly recognized that they play important roles in human health and disease. A recent review by Sepich-Poore and colleagues (1) provides a critical and comprehensive overview of current knowledge regarding the implications of the human microbiome in the origin and development of cancer, as well as its diagnostic and therapeutic potential. The idea that microbes can promote the origin of tumors is old, and acquired certain popularity in the late 1800s and early 1900s in the context of contentious findings that were later disproved. This led to a period of certain neglect of the microbial roles in cancer, and a focus on the genetic and environmental causes of cancer. Currently, thanks to decades of thorough study and new tools such as high throughput sequencing and meta-genomics, microbes have made a comeback to the cancer stage, and a more balanced view is emerging. Although some microbes have been directly associated to the origin of some cancers, the list of such "oncomicrobes" is rather limited with only 11 species labeled as such by the International Association of Cancer registries (2). Nevertheless, these carcinogenic microbes account for an estimated 2.2 million cases each year (a non-negligible $13 \%$ of the total). Beyond these direct implications, the human microbiome can play several indirect roles of a likely much larger impact in cancer. These indirect roles exerted by so-called "complicit" microbes can either promote or inhibit cancer development and are mediated by intricate relationships between microbial communities and the relevant tissue, including the infiltrated immune cells. The authors distinguish two main ways by which microbes influence the origin and progression of cancer. One, known as genotoxin-mediated mutagenesis, involves the direct mutational impact on human cells of microbial toxins, enzymes or other molecules, including reactive oxygen species, DNase, colibactin and other damaging toxins. This mutagenic effect of some microbial species is revealed by the presence of specific mutational signatures associated with the presence of specific species or strains in some cancer types such as colorectal, head and neck, and urinary cancers. Such genotoxic mechanisms would provide a direct connection between mutational signatures caused by microbes and somatic mutations known to be associated to tumors. Other major group of mechanisms, referred to as immunomodulation, involves modulation of the immune system by the microbes or their metabolic products. The immune system rarely initiates the cancer lesion, but can increase the progression of tumors through cronic imflamation or defective immunosurveyance, which in turn can be driven by the presence of certain microbes or their metabolic byproducts. This may lead to complex interactions of varying outcomes, such as the finding that common p53 mutations are carcinogenic in the presence of microbially produced gallic acid, but protective otherwise (3). Finally, microbes actively participate in the biotransformation of xenobiotics, including cancer drugs, thus interfering with cancer treatment in ways that are not 
fully understood. The authors rightly discuss the many difficulties of studying these complicit roles of microbes, which can act distally or proximally with respect to the tumor location. One outstanding question is whether microbes reside within tumors, which has been explored using DNA amplification and sequencing techniques (4), but which often remain to be corroborated by orthogonal techniques such as microscopy or probe hybridization. According to these results, resident bacteria are not rare in tumors, which calls for the necessity of studying the intimate relationships between resident bacteria, tumors and the immune system. The review provides a thorough revision of known relationships of cancer with the gut microbiome, which is by far the most studied niche. In this section, specific roles of gut microbes in the activation of the immune system, in driving chronic inflammation, or in metabolizing drugs are discussed, always providing clear illustrative examples. Finally, the diagnostic and therapeutic potential of microbial-cancer associations is discussed providing a forward-looking balanced discussion. Associations between the presence of certain microbes, or their metabolites, and the risk of cancer development, the presence of cancer or of features of cancer progression can always be exploited for diagnostic or prognostic purposes, even if the bases of these relationships are not understood. The relative affordability of targeted metagenomic or metabolomic testing entails a high diagnostic potential which is already progressing in certain types of cancer, such as colorectal cancer (5). Moving from a diagnostic potential to a therapeutic one involves a much deeper comprehension of the system that is targeted for interference. But microbial communities are actionable through different means, from the use of antibiotics or pre- and pro-biotics to the direct transplantation of microbial communities. In addition, molecular mimicry between cancer and microbial antigens could be exploited to treat cancer. Finally, experiments have shown that infiltration of bacteria resulted in most of the bacterial cells ending up in the tumor, which suggest that engineered bacteria could be used to deliver toxins to treat tumors (6). All these strategies open diverse possibilities for innovative treatments, but the authors cautiously remind that much research is needed before we can see some of these therapies in the clinics. Although, reflecting current research focus, the article stresses the roles of the gut microbiome, similar processes and relationships are expected for other niches such as the oral cavity (7), or the respiratory tract (8), among others. Altogether, the discussed review provides a very comprehensive and balanced overview of current knowledge on the roles of the human microbiome in cancer, including its diagnostic and therapeutic potential.

\section{Acknowledgments}

Funding: None.

\section{Footnote}

Provenance and Peer Review: This article was commissioned by the editorial office of Hepatobiliary Surgery and Nutrition. The article did not undergo external peer review.

Conflicts of Interest: The author has completed the ICMJE uniform disclosure form (available at https://hbsn. amegroups.com/article/view/10.21037/hbsn-21-241/coif). The author has no conflicts of interest to declare.

Ethical Statement: The author is accountable for all aspects of the work in ensuring that questions related to the accuracy or integrity of any part of the work are appropriately investigated and resolved.

Open Access Statement: This is an Open Access article distributed in accordance with the Creative Commons Attribution-NonCommercial-NoDerivs 4.0 International License (CC BY-NC-ND 4.0), which permits the noncommercial replication and distribution of the article with the strict proviso that no changes or edits are made and the original work is properly cited (including links to both the formal publication through the relevant DOI and the license). See: https://creativecommons.org/licenses/by-nc-nd/4.0/.

\section{References}

1. Sepich-Poore GD, Zitvogel L, Straussman R, et al. The microbiome and human cancer. Science 2021;371:eabc4552.

2. IARC Working Group on the Evaluation of Carcinogenic Risks to Humans. Biological agents. Volume 100 B. A review of human carcinogens. IARC Monogr Eval Carcinog Risks Hum 2012;100:1-441.

3. Rubinstein MR, Wang X, Liu W, et al. Fusobacterium nucleatum promotes colorectal carcinogenesis by modulating E-cadherin/ $\beta$-catenin signaling via its FadA adhesin. Cell Host Microbe 2013;14:195-206.

4. Nejman D, Livyatan I, Fuks G, et al. The human 
tumor microbiome is composed of tumor type-specific intracellular bacteria. Science 2020;368:973-80.

5. Saus E, Iraola-Guzmán S, Willis JR, et al. Microbiome and colorectal cancer: Roles in carcinogenesis and clinical potential. Mol Aspects Med. 2019;69:93-106.

6. Charbonneau MR, Isabella VM, Li N, et al. Developing a new class of engineered live bacterial therapeutics to treat human diseases. Nat Commun 2020;11:1738.

7. Willis JR, Gabaldón T. The Human Oral Microbiome in Health and Disease: From Sequences to Ecosystems. Microorganisms 2020;8:308.

8. Man WH, de Steenhuijsen Piters WA, Bogaert D. The microbiota of the respiratory tract: gatekeeper to respiratory health. Nat Rev Microbiol 2017;15:259-70.

Cite this article as: Gabaldón T. Roles of the human microbiome in cancer. HepatoBiliary Surg Nutr 2021;10(4):558560. doi: 10.21037/hbsn-21-241 